\title{
ARTICLE
}

\section{Reduced EGFR signaling enhances cartilage destruction in a mouse osteoarthritis model}

\author{
Xianrong Zhang ${ }^{1,2}$, Ji Zhu ${ }^{1}$, Fei Liu ${ }^{1,3}$, Yumei Li ${ }^{1,4}$, Abhishek Chandra ${ }^{1}$, L Scott Levin ${ }^{1}$, Frank Beier ${ }^{5}$, \\ Motomi Enomoto-Iwamoto ${ }^{1,6}$ and Ling Qin ${ }^{1}$
}

Osteoarthritis (OA) is a degenerative joint disease and a major cause of pain and disability in older adults. We have previously identified epidermal growth factor receptor (EGFR) signaling as an important regulator of cartilage matrix degradation during epiphyseal cartilage development. To study its function in OA progression, we performed surgical destabilization of the medial meniscus (DMM) to induce OA in two mouse models with reduced EGFR activity, one with genetic modification $\left(E g f r^{W a 5 /+}\right.$ mice) and the other one with pharmacological inhibition (gefitinib treatment). Histological analyses and scoring at 3 months post-surgery revealed increased cartilage destruction and accelerated OA progression in both mouse models. TUNEL staining demonstrated that EGFR signaling protects chondrocytes from OA-induced apoptosis, which was further confirmed in primary chondrocyte culture. Immunohistochemistry showed increased aggrecan degradation in these mouse models, which coincides with elevated amounts of ADAMTS5 and matrix metalloproteinase 13 (MMP13), the principle proteinases responsible for aggrecan degradation, in the articular cartilage after DMM surgery. Furthermore, hypoxia-inducible factor $2 \alpha$ (HIF2 $\alpha$ ), a critical catabolic transcription factor stimulating MMP13 expression during OA, was also upregulated in mice with reduced EGFR signaling. Taken together, our findings demonstrate a primarily protective role of EGFR during OA progression by regulating chondrocyte survival and cartilage degradation.

Bone Research (2014) 2, 14015; doi:10.1038/boneres.2014.15; published online: 05 August 2014

\section{INTRODUCTION}

Osteoarthritis (OA) is a progressive and degenerative disorder of the joint primarily characterized by the destruction of articular cartilage. Articular cartilage is predominantly composed of collagen, proteoglycans and other matrix proteins. Loss of large proteoglycan, aggrecan, decreases cartilage compressive stiffness and precedes the damage to the collagen fibrillar network, which is responsible for tensile properties of the tissue. The structural and functional integrity of articular cartilage relies on the balance between anabolic and catabolic activities of chondrocytes. The pathology of OA in cartilage involves the production of pro-inflammatory cytokines, inflammation, degradation of extracellular matrix (ECM) by proteases, chondrocyte hypertrophy and apoptosis.' Moreover, the subchondral bone is inevitably altered with cortical plate thickening, enhanced bone remodeling and osteophyte formation. ${ }^{2}$ The past decade has witnessed significant advances in deciphering the basic mechanism by which OA develops using genetically modified and surgical animal models, but there are as yet no disease modifying treatments available. It remains imperative to understand what drives cartilage degeneration during $O A$.

Growth factors, such as transforming growth factor beta (TGF- $\beta$ ), insulin-like growth factors, bone morphogenic proteins and fibroblast growth factors, regulate synthesis and maintenance of cartilage ECM and therefore, play important roles in cartilage homeostasis and OA development. We recently demonstrated that epidermal growth factor receptor (EGFR), a tyrosine kinase receptor, and one of its cognate ligands, TGF- $\alpha$, are important for

'Department of Orthopaedic Surgery, School of Medicine, University of Pennsylvania, Philadelphia, PA, USA; '²epartment of Physiology, School of Basic Medical Sciences, Wuhan University, Wuhan, China; ${ }^{3}$ Department of Plastic and Reconstructive Surgery, Shanghai Ninth People's Hospital, Shanghai, China; ${ }^{4}$ Shanghai Municipal Hospital of Traditional Chinese Medicine, Shanghai University of Traditional Chinese Medicine, Shanghai, China; ${ }^{5}$ Department of Physiology and Pharmacology, Schulich School of Medicine and Dentistry, University of Western Ontario, London, ON, Canada and ${ }^{6}$ Department of Surgery, The Children's Hospital of Philadelphia, Philadelphia, PA, USA

Correspondence: L Qin (mailto:qinling@mail.med.upenn.edu)

Received: 6 April 2014; Revised: 9 June 2014; Accepted: 12 June 2014 
cartilage matrix degradation during endochondral ossification. ${ }^{3-4}$ Deficiency of EGFR activity either globally or specifically in chondrocytes causes expansion of the hypertrophic zone in the growth plate ${ }^{3,5}$ and delayed formation of secondary ossification center in long bones at early postnatal stage. ${ }^{6}$ Consistently, TGF- $\alpha$ null mice exhibited similar developmental phenotypes. ${ }^{4}$ Mechanistic studies revealed that activation of EGFR signaling stimulates the expression of matrix metalloproteinases (MMPs), including MMP9, 13 and 14, and receptor activator of nuclear factor-kB ligand (RANKL) in hypertrophic chondrocytes via $\beta$-catenin-dependent and -independent pathways, resulting in increased cartilage matrix degradation by chondrocytes and osteoclasts at the chondroosseous junctions. ${ }^{6}$ EGFR ligands are membrane-bound proteins requiring ectodomain shedding to release the functional peptides. The major sheddase for TGF- $\alpha$ is TNF$\alpha$-converting enzyme, also known as a disintegrin and metalloprotease 17 (ADAM17). ${ }^{7}$ Studies of cartilage-specific TNF- $\alpha$-converting enzyme knockout mice revealed a similar growth plate phenotype, providing further evidence that EGFR signaling stimulates the degradation of cartilage matrix. ${ }^{8-9}$

Since cartilage ECM degradation is a critical step toward OA pathogenesis, we hypothesized that EGFR and its ligands constitute another growth factor signaling pathway regulating OA development. Indeed, previous reports implied that this pathway might play a catabolic role in OA pathology. It was reported that the amount of TGF- $\alpha$ is elevated by articular chondrocytes in experimentally induced OA and human OA. ${ }^{10-11}$ Disruption of mitogen-inducible gene 6 (mig-6), a negative feedback inhibitor of EGFR activity, resulted in a degenerative joint OA-like disease in mice. ${ }^{12-14}$ Moreover, mice deficient in MMP13 activity either globally ${ }^{15}$ or conditionally in chondrocytes $^{16}$ were protected from OA development induced by surgery. In the present study, in order to directly investigate the role of EGFR in OA development, we performed destabilization of the medial meniscus (DMM) to induce $O A$ in mouse models with reduced EGFR activity. This OA surgery is well characterized and it induces progressive cartilage degeneration with little synovial inflammation. ${ }^{17}$ Surprisingly, we found that these mice show more severe cartilage destruction compared to control mice. Furthermore, histological analyses revealed that loss of EGFR activity accelerates chondrocyte apoptosis and increases cartilage matrix degradation, which coincide with the upregulation of proteinases (ADAMTS5 and MMP13), and a catabolic transcription factor, HIF2 $\alpha$. These results uncover an important function of EGFR in protecting articular cartilage during OA progress and suggest this protein as a potential target for OA treatment.

\section{MATERIALS AND METHODS}

Surgical induction of OA by DMM in mice with deficient EGFR activity

Two mouse models with deficient EGFR activity were used in our study. In the first model, mice were heterozygous for a dominant negative allele of EGFR, Wa5. ${ }^{18}$ These mice (Egfr $^{\mathrm{Wa} 5 /+}$ ) on a 129S1/SvImJ background were generated by breeding Egfr ${ }^{W a 5 /+}$ and wild-type (WT, Egfr ${ }^{+/+}$) mice, and were identified by their wavy hair appearance. Both EgfrWa5/+ $(n=9)$ and their WT siblings $(n=10)$ at 3 months of age were subjected to DMM surgery as detailed below. In the second model, WT 129 S2 mice were purchased from Charles River (Malvern, PA, USA) at the age of 2 months. A month later, they received DMM surgery followed by either vehicle $10.5 \%$ methyl cellulose, $\mathrm{n}=8$ ) or gefitinib (100 $\mathrm{mg} \cdot \mathrm{kg}^{-1}, \mathrm{n}=9$, an EGFR inhibitor; LC Laboratories, Woburn, MA, USA) treatment via oral gavage immediately after surgery, once every other day, for 12 weeks. In both models, there was no significant difference in body weight between control and EGFR deficient group at the beginning of surgery and at 3 months after surgery when the knee joints were harvested.

Experimental OA was induced by DMM surgery and performed by a single surgeon (XZ) in these two mouse models as described previously. ${ }^{17}$ Only male mice were used in our study because male 129 mice develop more severe OA than females. ${ }^{19}$ Briefly, under general anesthesia, the right knee joint capsule was exposed and the medial meniscotibial ligament was transected under microscope to give destabilization of the medial meniscus. A sham operation was performed on the left knee joint in which the ligament was visualized but not transected. Mice were maintained in their preoperative groups, allowed unrestricted cage exercise, and were weighed weekly until they were euthanized 12 weeks after surgery. All animal works performed in this study were approved by the Institutional Animal Care and Use Committee at the University of Pennsylvania.

Histological analysis of OA cartilage

After euthanasia, bilateral knee joints were harvested free of soft tissues, fixed in $4 \%$ paraformaldehyde overnight, decalcified in $0.5 \mathrm{~mol} \cdot \mathrm{L}^{-1}$ ethylene diamine tetraacetic acid (EDTA) ( $\mathrm{pH} 7.4$ ) for 4 weeks at $4{ }^{\circ} \mathrm{C}$ and embedded in paraffin. Serial $5-\mu \mathrm{m}$-thick sagittal sections were cut across the medial compartment of the joint. Two sections within every consecutive six sections were stained with Safranin O/Fast green and hematoxylin and the one with better morphology was used for quantification. Each knee yielded about 15 sections for scoring by two blinded observers (XZ and ME-I) using modified Mankin's methods. ${ }^{20}$ In brief, each section was assigned a score, which is the sum of cartilage structure (0-5), chondrocytes (0-3), SafraninO staining (0-5) and tidemark (0-1). Each knee received a 
score from the section with the maximal score. The results of Mankin score represent the mean of maximal scores in each group. To characterize the loss of articular cartilage, cartilage area (total) and Safranin O-stained area (uncalcified) were outlined and quantified on projected images of about 15 sections per bone selected as above followed by averaging. Thickness of each area was determined by averaging five thicknesses evenly distributed across the entire cartilage. To quantify changes in hypertrophic chondrocytes in the shamoperated knees, the number of hypertrophic chondrocytes in tibial articular cartilage was counted based on their morphology (enlarged chondrocyte lacunae with a lack of Safranin-O stain around a collapsed cells) and divided by articular cartilage area.

Immunohistochemistry and TUNEL staining

Serial sections adjacent to those with representative Safranin O-stained images were dewaxed in xylene and rehydrated through a graded series of alcohols for immunohistochemical evaluation. Briefly, after antigen retrieval with boiling in sodium citrate buffer, endogenous peroxidase activity was quenched in $3 \% \mathrm{H}_{2} \mathrm{O}_{2}$ for 15 min and washed in phosphate buffered saline (PBS). Sections were then blocked in serum for $30 \mathrm{~min}$ followed by incubation with the primary antibody in humidified chamber at $4{ }^{\circ} \mathrm{C}$ overnight. Biotinylated secondary antibody was added for $30 \mathrm{~min}$ on the second day, followed by an avidin-biotinylated horseradish peroxidase complex according to the manufacturer's directions (Vectastain $A B C$ Kit; Vector Laboratories, Burlingame, CA, USA). Finally, peroxidase activity was revealed by immersion in DAB substrate (Dako, Glostrup, Denmark). The following primary antibodies were used: rabbit anti-aggrecanase generatedaggrecan neoepitope NITEGE, rabbit anti-MMP-generated aggrecan-neoepitope DIPEN (gifts from Dr John S Mort), rabbit anti-MMP13 (ab75606; Abcam, Cambridge, MA, USA), rabbit anti-ADAMTS5 (ab41037; Abcam), rabbit anti-HIF2 $\alpha$ (NB100-122; Novus Biologicals, Littleton, CO, USA). TUNEL staining was carried out on paraffin sections using ApopTag Plus Peroxidase in Situ Apoptosis Detection Kit (S7101; Millipore, Billerica, MA, USA) according to the manufacturer's instructions.

Primary chondrocytes, QRT-PCR and apoptosis assay Primary epiphyseal chondrocytes were obtained from rat newborn pups as described previously. ${ }^{3}$ Cells were plated at a density of $4 \times 10^{4}$ per $\mathrm{cm}^{2}$ in 12-well plates in chondrogenic medium (DMEM/F12 medium with $5 \%$ fetal bovine serum, $50 \mu \mathrm{g} \cdot \mathrm{mL}^{-1}$ of L-ascorbic acid, $1 \%$ glutamine, $100 \mu \mathrm{g} \cdot \mathrm{mL}^{-1}$ of streptomycin and $100 \mathrm{U} \cdot \mathrm{mL}^{-1}$ of penicillin). Four days later, when cells reached $90 \%$ confluence, cultures were given fresh medium overnight before adding
$50 \mathrm{ng} \cdot \mathrm{mL}^{-1}$ of recombinant human TGF- $\alpha$ (Pepro-Tech, Rocky Hill, NJ, USA). RNA was harvested after $48 \mathrm{~h}$ for QRT-PCR. The primer sequences are: adamts5 (forward 5'-CGTTCCTGCAGTGTCATACCCT-3', reverse 5'-TTTGACTCCTTTTGCATCGGAC-3') and hif2 $\alpha$ (forward 5'-GTGGTCTGTGGGCAATCAGAGCG-3', reverse 5'-GGAGACATGAGGCGGGGTGC-3').

To study the survival effect of TGF- $\alpha$, primary rat chondrocytes were seeded in 12-well plates at $4 \times 10^{4}$ cells per $\mathrm{cm}^{2}$ and serum-starved overnight. The next day, cells were pretreated with either vehicle or $25 \mathrm{ng} \cdot \mathrm{mL}^{-1}$ tumor necrosis factor $\alpha$ (TNF- $\alpha$ ) (Pepro-Tech) before the addition of $50 \mathrm{ng} \cdot \mathrm{mL}^{-1}$ TGF- $\alpha$. Two days later, cells were washed with PBS and stained with $5 \mu \mathrm{g} \cdot \mathrm{mL}^{-1}$ ethidium bromide and $5 \mu \mathrm{g} \cdot \mathrm{mL}^{-1}$ acridine orange in $1 \times \mathrm{PBS}$ as described previously. ${ }^{21}$ Apoptotic cells were identified as cells having condensed chromatin (green at early apoptotic stage and red at late apoptotic stage). Living cells had a characteristic green chromatin staining with a normal morphology of the nucleus. The number of apoptotic and total cells was counted in three fields per well under a fluorescence microscope.

\section{Statistic analysis}

All statistical analysis was performed by independent Student's t-test assuming equal variances in each group or two-way analysis of variance with a Bonferroni's post hoc test. For cell culture experiments, all results are derived from experiments being repeated independently at least three times. A value of $P<0.05$ was considered significant. All data are expressed as mean \pm s.e.m.

\section{RESULTS}

Mice with reduced EGFR activity exhibit accelerated OA development

Two mouse models with reduced EGFR activity were studied. In the first model, we compared OA development between Egfr ${ }^{\text {wa5/+ }}$ and their WT siblings after DMM surgery. Egfrwa5 codes for a kinase-dead, dominant negative receptor. Mice homozygous for Wa5 are embryonic lethal, but the heterozygotes are viable and show no major pathological changes, ${ }^{18}$ allowing for long-term studies. We recently reported that primary chondrocytes derived from Egfr ${ }^{\mathrm{wa} 5 /+}$ mice shows a slightly decrease in extracellular signal-regulated kinases (ERK) phosphorylation after being treated with epidermal growth factor (EGF) compared to WT cells, suggesting that the EGFR activity in chondrocytes from Egfr ${ }^{\text {wa5/+ }}$ mice is modestly suppressed. ${ }^{6}$ In the second model, we compared OA development in WT mice treated with either vehicle or gefitinib, an EGFR kinase inhibitor. ${ }^{22}$ Previously, we have demonstrated that similar inhibitor treatment results significant bone loss at both trabecular and cortical sites in young mice. ${ }^{23}$ 
DMM surgery was performed in the right knees of 3month-old skeletally mature mice in both models to induce OA. The left knees received sham operation. Three months after surgery, knee joints were harvested for histological analyses. The sham-operated knees appeared normal in morphology and their Mankin scores representing the severity of OA were negligible (less than 1) in all groups (data not shown). In DMM-operated knees, while WT mice exhibited typical OA features, Egfr Wa5/+ mice had marked increases in cartilage surface fibrillation, clefting and erosion down to the tidemark (Figure la). Moreover, total cartilage area and thickness, particularly

a

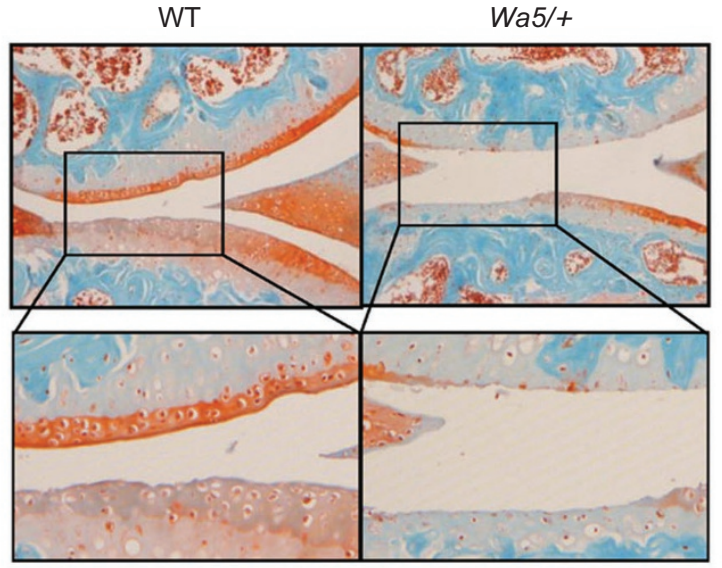

b

Femoral condyle

Tibial plateau
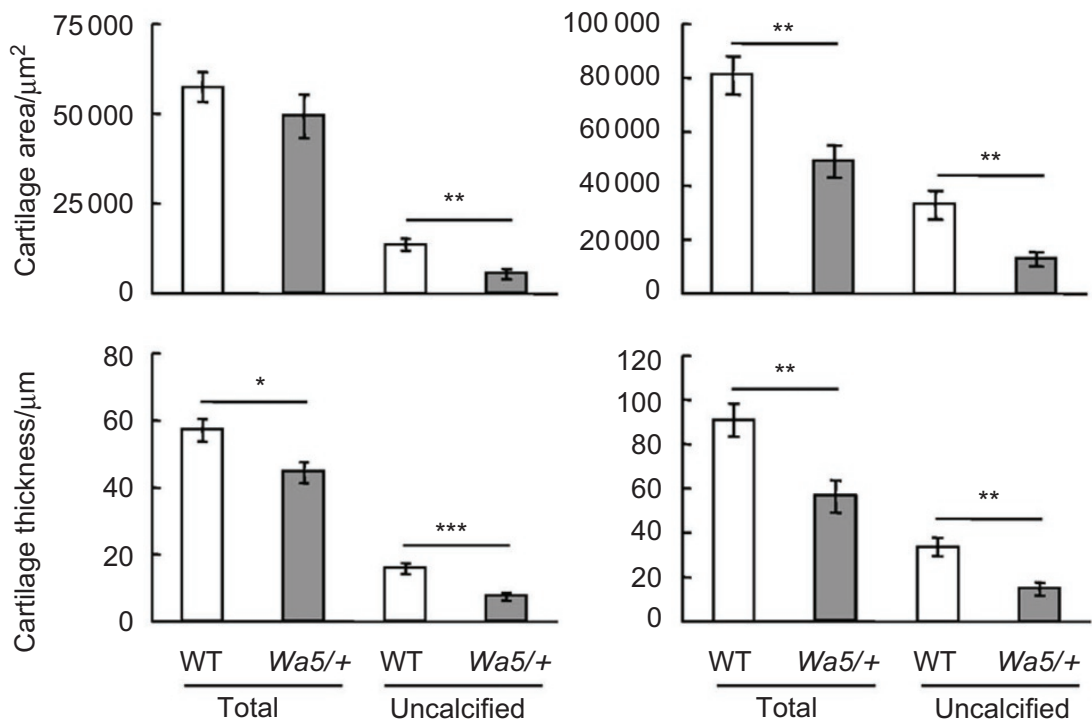

c

Femoral condyle

Tibial plateau
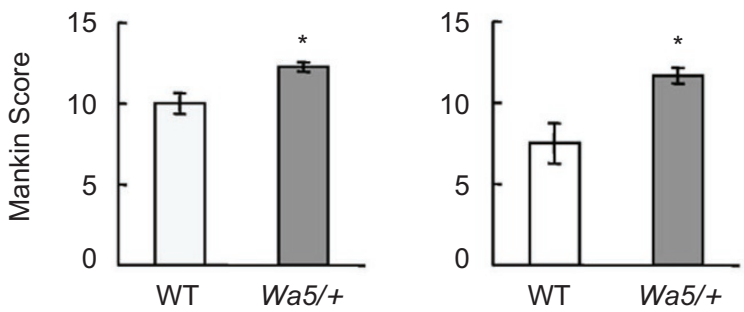

Figure 1. Egfr $\mathrm{Wa5} /+_{+}$mice exhibit accelerated osteoarthritis progression after DMM surgery. (a) Representative Safranin O/Fast Green staining images of mouse knee joints show increased articular cartilage degradation in $E g f r^{W a 5 /+}$ mice 3 months after DMM surgery. Bottom panels are magnified images of top panels. (b) Total articular cartilage, uncalcified articular cartilage, and their respective thickness were quantified at both femoral condyle and tibial plateau regions. (c) Mankin score graded by blinded observers confirmed more articular cartilage destruction in $E g f r$ Wa5/+ mice $(n=9)$ compared to WT $(n=10)$ mice after DMM surgery. ${ }^{*} P<0.05 ; * * P<0.01 ; * * * P<0.001$. 
the uncalcified area and thickness, at both medial femoral condyle and medial tibial plateau were reduced in Egfr Wa5/+ mice in comparison with WT mice (Figure 1b). Further scoring by Mankin's method revealed significant $19 \%$ and $36 \%$ increases of OA scores at medial femoral condyle and medial tibial plateau, respectively, in Egfr $^{\text {Wa5/+ }}$ mice compared to those in WT mice (Figure 1C).

In gefitinib-treated mice, OA damages on the articular cartilage was relatively milder than those in $\mathrm{Egfr}^{\mathrm{Wa} /+}$ mice, but still more severe than their vehicle-treated controls with increased fibrillation, clefting and erosion at the surface of articular cartilage (Figure 2a). While we did not observe overall loss of total articular cartilage in gefitinib-treated mice, the area and thickness of uncalcified cartilage were remarkably decreased in those mice compared to their vehicle controls (Figure 2b). Mankin scores revealed a significant $28 \%$ increase at medial femoral condyle and a trend of $22 \%$ increase at medial tibial plateau in these mice compared to controls (Figure 2c). Taken together, these results demonstrate that mice with

a

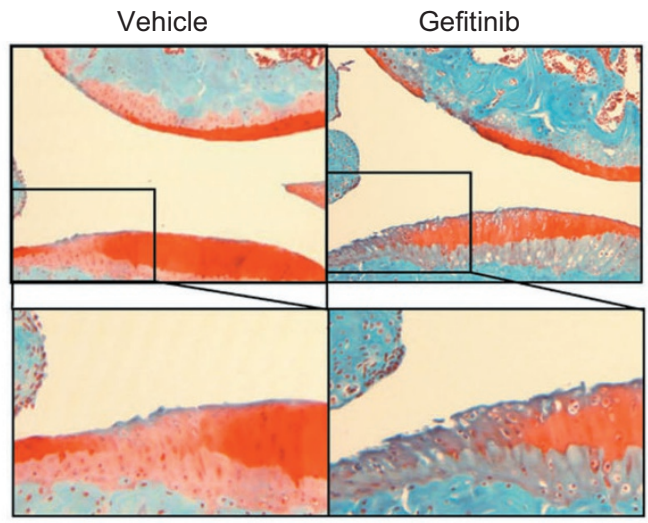

b
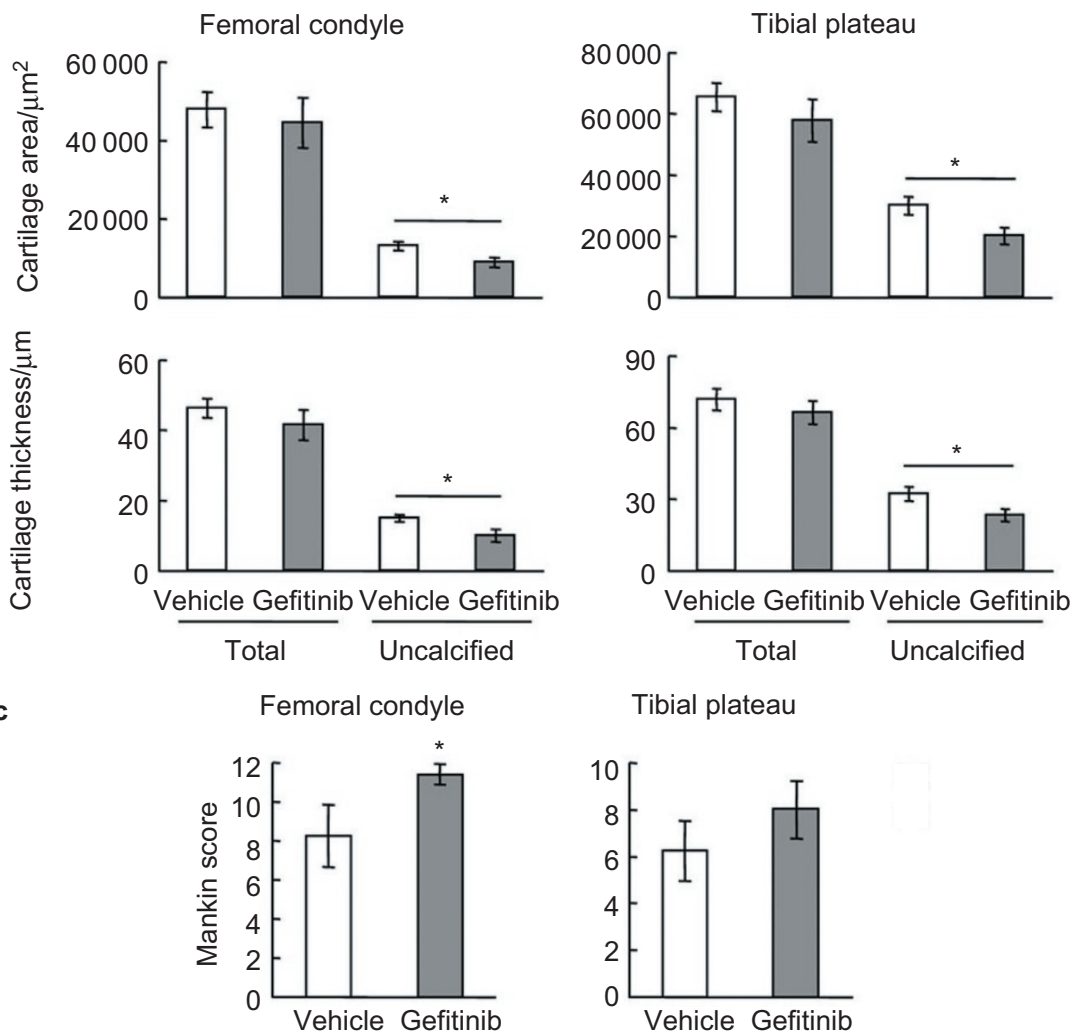

Tibial plateau

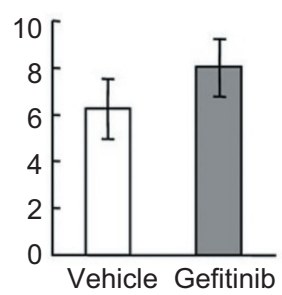

Figure 2. Mice treated with gefitinib exhibit accelerated osteoarthritis progression after DMM surgery. (a) Representative Safranin O/Fast Green staining images of mouse knee joints show increased articular cartilage degradation in gefitinib-treated mice 3 months after DMM surgery. Bottom panels are magnified images of top panels. (b) Total articular cartilage, uncalcified articular cartilage, and their respective thickness were quantified at both femoral condyle and tibial plateau regions. (c) Mankin score graded by blinded observers confirmed more articular cartilage destruction in gefitinib-treated mice $(n=9)$ compared to vehicle-treated $(n=8)$ mice after DMM surgery. $* P<0.05$. 
reduced EGFR activity exhibit more cartilage destruction and accelerated OA progression.

Chondrocyte hypertrophy is routinely seen in the development of osteoarthritis. Since our previous work demonstrated that EGFR deficiency induces expansion of the hypertrophic zone of the growth plate in young rats and neonatal mice, ${ }^{3}$ we quantified the number of hypertrophic chondrocytes in the articular cartilage and normalized it against the total cartilage area. In the sham-operated contralateral joints, we observed a significant $111 \%$ increase of hypertrophic chondrocytes in Egfr Wa5/+ mice (WT: $0.12 \pm 0.03$ cells per $\mathrm{mm}^{2}$; Egfr ${ }^{\mathrm{Wa} 5 /+}$ : $0.25 \pm 0.02$ cells per $\mathrm{mm}^{2}, n=5$ in each group, $P=0.01$, Figure $3 a$ ) and a similar trend of increase in gefitinib-treated mice (vehicle-treated: $0.19 \pm 0.02$ cells per $\mathrm{mm}^{2}$; gefitinib-treated: $0.24 \pm 0.03$ cells per $\mathrm{mm}^{2}, n=5$ in each group, $P=0.17$, Figure $3 b$ ) compared to their respective controls. We found that it was difficult to perform similar measurement at the DMM knees because of severe erosion and/or loss of articular cartilage in the EGFR deficient group. These findings indicate that EGFR prevents chondrocyte hypertrophy and may explain why mice with reduced EGFR signaling are predisposed to the development of osteoarthritis.

Reduced EGFR activity promotes apoptosis in cartilage chondrocytes after DMM surgery

Hypertrophic chondrocytes undergo cell death and are replaced by bone in the process of endochondral ossification. However, this process at the articular surface could lead to osteoarthritis. Chondrocyte apoptosis has been observed during OA progression and the apoptotic rate is positively correlated with the severity of cartilage damage. ${ }^{24-25}$ Indeed, the contribution of apoptosis to OA was further demonstrated by using caspase inhibitors, which block cell death and decrease severity of cartilage lesions in posttraumatic OA. ${ }^{26}$ EGFR has multifaceted actions on cells from a variety of tissues and is capable of regulating their proliferation, differentiation, survival, migration, and adhesion. To investigate the role of EGFR in chondrocyte apoptosis during OA development, TUNEL staining was performed on knee joints at 3 months post-surgery in mouse models with reduced EGFR activity. In DMM-operated knees from control mice, only $8 \%-12 \%$ of chondrocytes in articular cartilage underwent apoptosis and most of these apoptotic chondrocytes were located in the calcified zone (Figure $4 \mathrm{a}$ and $4 \mathrm{~b}$ ). In contrast, $30 \%$ and $24 \%$ of chondrocytes in DMM-operated knees from Egfr ${ }^{\text {wa5/+ }}$ mice and gefitinib-treated mice, respectively, were TUNEL-positive and many of them extended into the middle and deep zones (Figure $4 a$ and $4 b$ ), suggesting that EGFR protects chondrocytes from OA-induced cell death.

To confirm this finding in vitro, we isolated epiphyseal chondrocytes from neonatal pups and used serum starvation together with TNF- $\alpha$, a pro-inflammatory cytokine and a well-known inducer of chondrocyte apoptosis in $\mathrm{OA}^{27}$ to induce cell death in these cells. Ethidium bromide/acridine orange staining showed that removal of serum alone increased the percentage of apoptotic cells from $2 \%$ to $14 \%$ and that addition of TNF- $\alpha$ further elevated it to $21 \%$ (Figure 4c). However, cotreatment with TGF- $\alpha$ completely abrogated the apoptotic signals of serum depletion and TNF- $\alpha$, reducing the percentage of apoptotic cells to the level similar to those grown in normal serum-containing medium (Figure 4c). Taken together, these in vivo and in vitro results clearly indicate that EGFR transduces survival signals to chondrocytes during OA progression.

Aggrecan degradation is accelerated in OA knee joints from mice with reduced EGFR activity

Proteolytic cleavage of aggrecan weakens the cartilage matrix and is a key event in OA pathogenesis. ${ }^{28-29} \mathrm{Next}$, we examined aggrecan degradation products in articular cartilage after DMM surgery by immunohistochemistry.

a

WT

Wa5/+

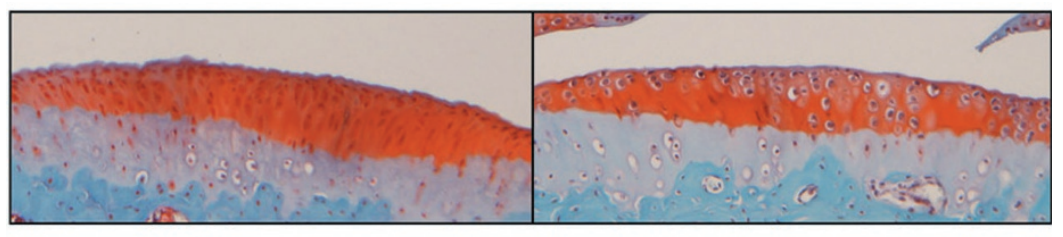

b

Vehicle

Gefitinib

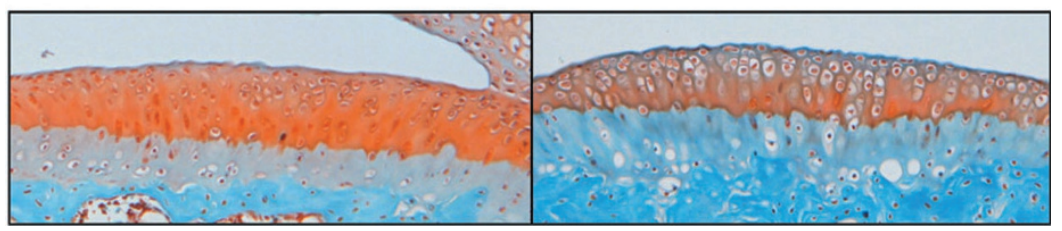

Figure 3. Mice with reduced EGFR activity have more hypertrophic chondrocytes in the articular cartilage. Representative Safranin O/Fast Green staining images of sham-operated tibial plateau from $E_{g} f_{r}{ }^{\mathrm{Wa} /+}$ mice (a) and gefitinib-treated mice (b) and their respective controls. 
a

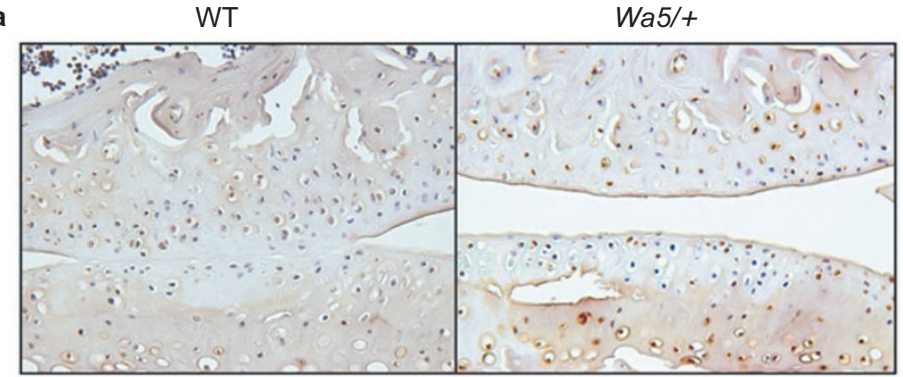

TUNEL ${ }^{+}$chondrocytes $/ \%$

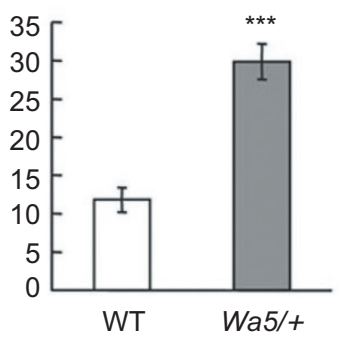

b

Vehicle

Gefitinib
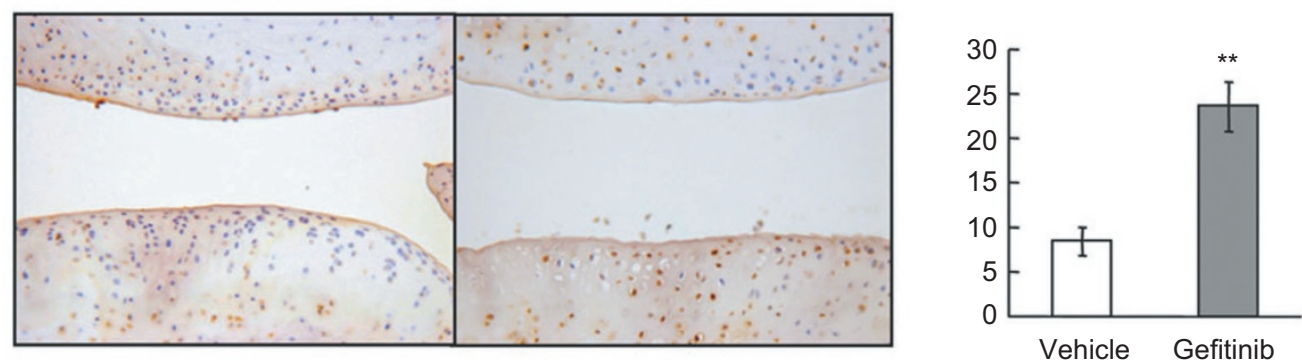

c

Serum starvation

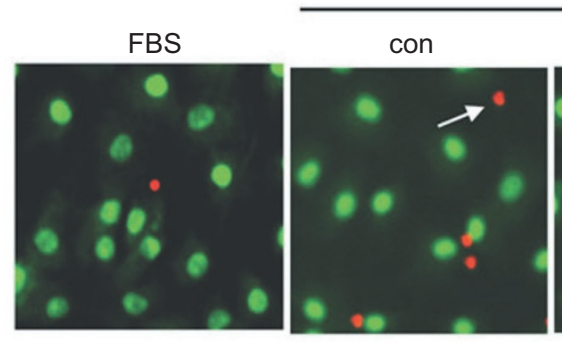

TGF $\alpha$

$\mathrm{TNF} \alpha$
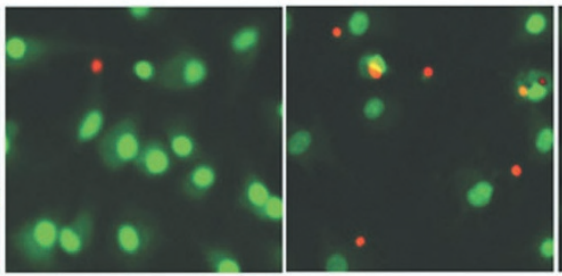

TNF $\alpha+T G F \alpha$

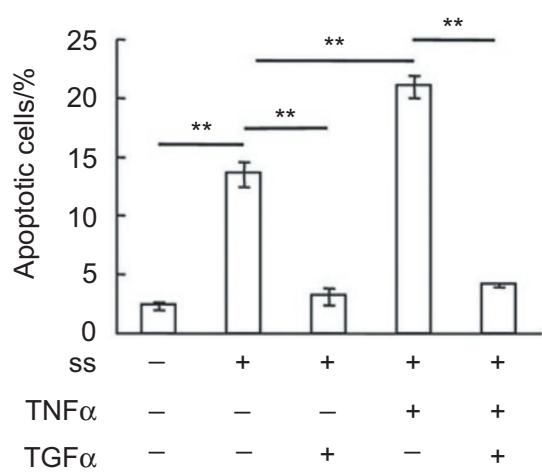

Figure 4. EGFR signaling protects articular chondrocytes from OA-induced apoptosis. (a) TUNEL staining showed increased number of apoptotic chondrocytes in knee joints from Egfr ${ }^{W a 5 /+}$ mice at 3 months post-surgery compared to that from WT mice. The percentage of TUNEL-positive chondrocytes was quantified ( $n=4$ in each group, right panel). ${ }^{\star \star \star} P<0.001$. (b) The same TUNEL staining was performed with gefitinib- and vehicletreated mice and quantification showed similar results ( $\mathrm{n}=4$ in each group, right panel). $* * P<0.01$. (c) TGF- $\alpha$, an EGFR ligand, suppresses chondrocyte apoptosis induced by serum depletion and TNF- $\alpha$. Primary chondrocytes were serum starved for 1 day followed by addition of $10 \%$ serum (FBS), vehicle (con), $50 \mathrm{ng} \cdot \mathrm{mL}^{-1} \mathrm{TGF}-\alpha, 25 \mathrm{ng} \cdot \mathrm{mL}^{-1} \mathrm{TNF}-\alpha$ and TNF- $\alpha$ plus TGF- $\alpha$. Two days later, ethidium bromide/acridine orange staining was performed to quantify the percentage of apoptotic cells (bottom panel). Arrow points to a late apoptotic cell. ss, serum starvation. ${ }^{* *} P<0.01$; *** $P<0.001$.

Staining of aggrecan-neoepitopes NITEGE (aggrecanasegenerated aggrecan cleavage fragment) and DIPEN (MMP-generated aggrecan cleavage fragment) showed that aggrecan degradation products generated by either aggrecanases or MMPs were significantly enhanced in both femoral and tibial articular cartilage areas in Egfr $^{\mathrm{Wa5} /+}$ mice and gefitinib-treated mice compared to their respective control mice (Figure 5). These results are in line with much more severe loss of Safranin O staining observed in knee joints of mice with reduced EGFR activity 


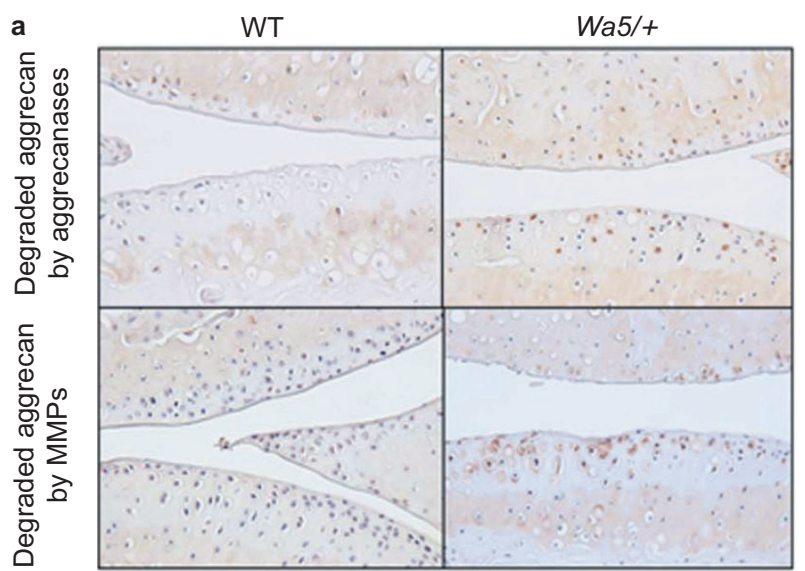

b

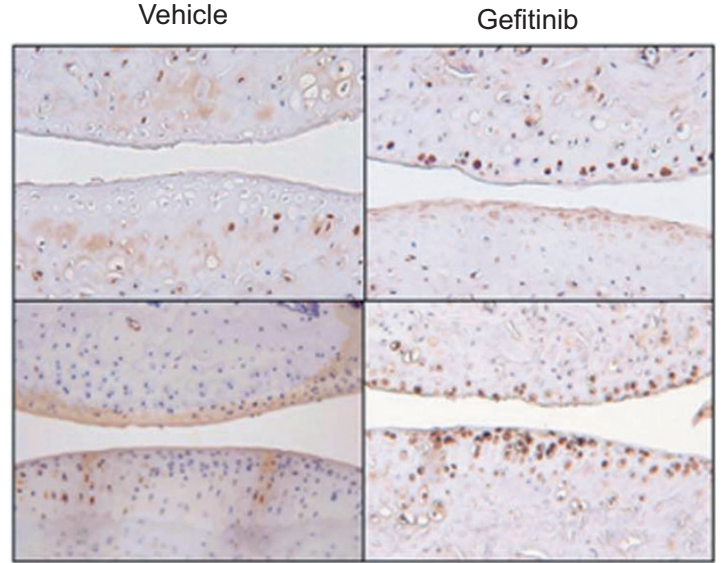

Figure 5. Aggrecan degradation is elevated in osteoarthritic cartilage from mice with reduced EGFR activity. Immunohistological assay revealed increased amounts of degraded aggrecan products generated either by aggrecanases (top panels) or by MMPs (bottom panels) in knee joints from WT and $E g f r^{W a 5 /+}$ mice (a) and vehicle- and gefitinib-treated mice (b) after DMM surgery.

(Figure $1 \mathrm{a}$ and $2 \mathrm{a}$ ), indicating that activation of EGFR signaling pathway suppresses cartilage matrix degradation during OA development.

ADAMTS5 is the major aggrecanase in mouse cartilage and deletion of this proteinase blocks cartilage degradation and prevents OA progression. ${ }^{30-31}$ Immunostaining revealed that ADAMTS5 amounts were markedly increased in articular cartilage after DMM surgery in both mouse models with reduced EGFR activity, compared to those in their respective control mice (Figure $6 \mathrm{a}$ and $6 \mathrm{~b}$, right panels). Similar observations were also obtained in sham-operated knees (Figure 6a and 6b, left panels), implying that EGFR signaling regulates adamts 5 expression in chondrocytes. Indeed, qRT-PCR experiment with primary chondrocytes further demonstrated that activation of EGFR in chondrocytes inhibits the mRNA expression of adamts5 (Figure 6c).

MMP13 is another critical proteinase responsible for cartilage degradation during OA development. ${ }^{15-16,32}$ Our previous work found that activation of EGFR signaling induces Mmp 13 expression in chondrocytes, while inhibition of EGFR signal by gefitinib attenuates its expression in the growth plate. ${ }^{3}$ Immunostaining of MMP13 was weak and mostly located below the tidemark in sham-operated knees. In line with the above growth plate results, we found that MMP13 amount was further reduced in articular cartilage from Egfr ${ }^{W a 5 /+}$ mice and gefitinib-treated mice (Figure $6 \mathrm{a}$ and $6 \mathrm{~b}$, left panels). In contrast, MMP13 immunostaining occurred in the uncalcified cartilage above the tide marker in DMM knee joints of Egfr Wa5/+ mice and gefitinib-treated mice and its amounts in those mice were significantly elevated compared to their respective control mice (Figure $6 a$ and $6 \mathrm{~b}$, right panels). Taken together, our results indicate that inhibition of EGFR signaling promotes aggrecan degradation during $O A$ development by increasing the amounts of important catabolic enzymes, such as ADAMTS5 and MMP13.

To understand the mechanism for the opposite regulation of Mmpl3 expression by EGFR in sham- and DMMoperated knee joints, we next analyzed the expression of hif2a, a transcription factor highly expressed in OA development and essential for $\mathrm{Mmp} 13$ expression in chondrocytes, ${ }^{33-35}$ in the articular cartilage of those mouse models after DMM surgery. Immunostaining revealed a considerably increased number of HIF2 $\alpha$-positive cells in articular cartilage from Egfr $\mathrm{Wa5/+}$ and gefitinib-treated mice at 12 weeks after surgery, compared to their respective control mice (Figure 7a and 7b). To confirm these in vivo results, we evaluated the regulation of HIF $2 \alpha$ expression by EGFR signaling in primary epiphyseal chondrocytes culture. QRT-PCR demonstrated that TGF- $\alpha$ significantly decreased hif $2 \alpha$ expression in primary chondrocytes (Figure 7c). Hence, our results suggest that one mechanism for EGFR to suppress $\mathrm{Mmp} 13$ expression is likely through inhibiting hif $2 \alpha$ expression during OA progression. In normal knees, since the basal level of HIF $2 \alpha$ is very low in the cartilage, ${ }^{34}$ EGFR signaling must use alternative transcription factor(s) to activate the expression of $\mathrm{Mmp} 13$.

\section{DISCUSSION}

The present study identifies a novel role of EGFR signaling in articular cartilage homeostasis and OA progression. We demonstrated that a genetically modified mouse model (Egfr ${ }^{\mathrm{Wa} /+}$ ) and a pharmacological mouse model (gefitinib treatment), both of which have reduced EGFR activity, exhibit accelerated articular cartilage destruction in knee joints after surgical induction of OA. This observation is correlated with increased chondrocyte apoptosis and aggrecan degradation. Further mechanistic studies suggest that this is likely due to augmented expression of 

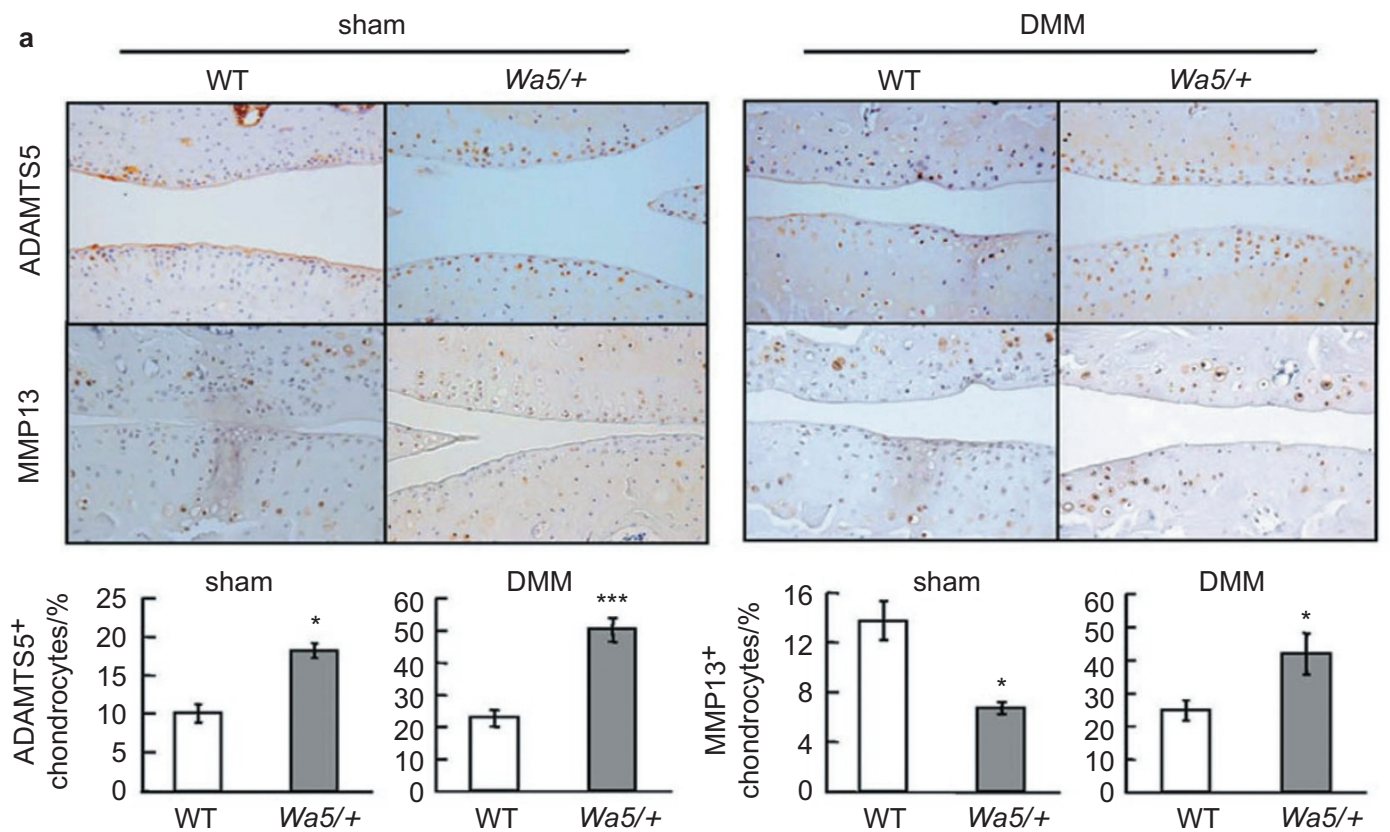

b
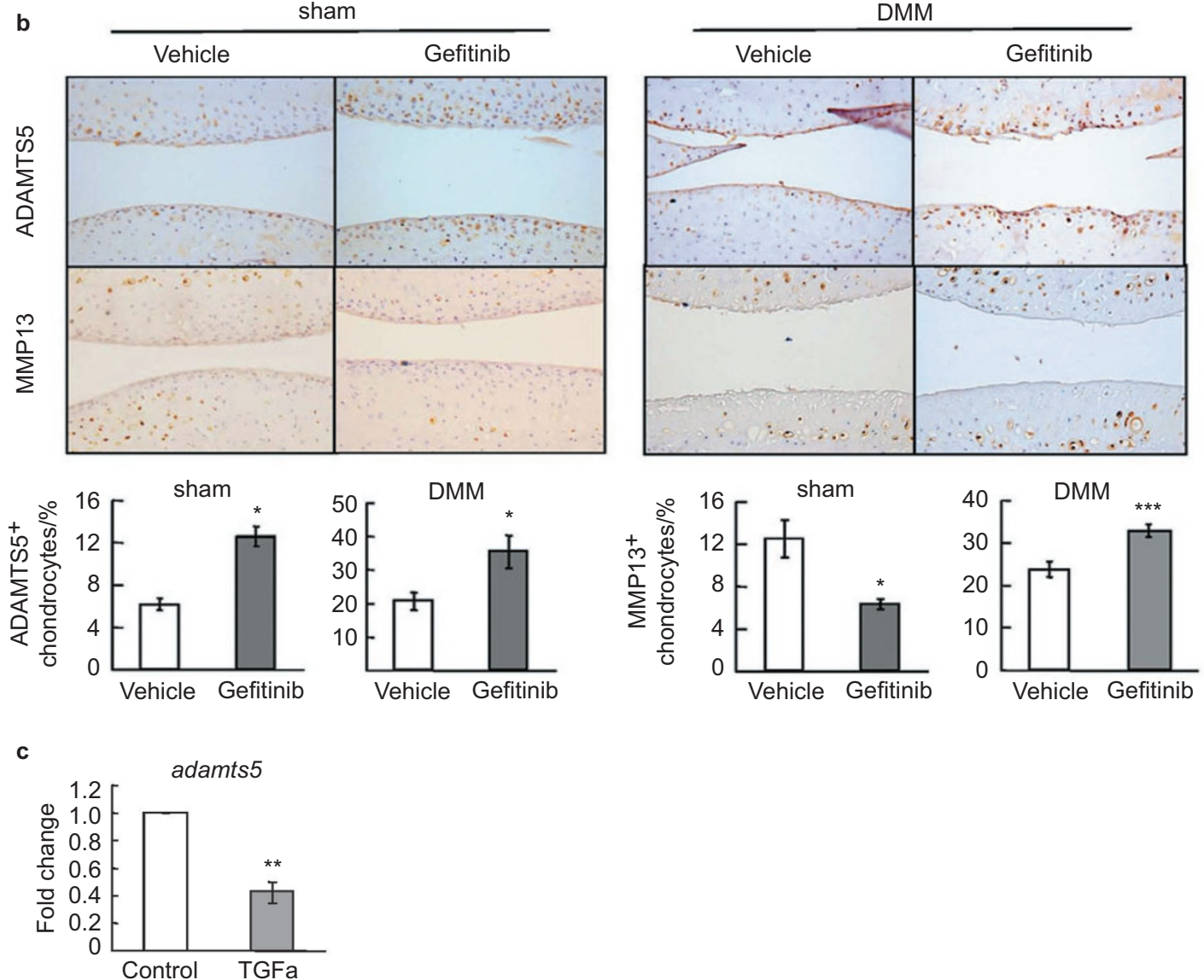

Figure 6. The protein amounts of ADAMTS5 and MMP13 are increased in osteoarthritic cartilage from mice with reduced EGFR activity. (a, b) Immunostaining of ADAMTS5 and MMP13 in sham- (left panels) and DMM-operated (right panels) knee joints from WT and Egfr Wa5/+ mice (a) and vehicle- and gefitinib-treated mice (b). For ADAMTS5, we observed increased staining in both sham and DMM knees from mice with reduced EGFR activity. However, for MMP13, we observed decreased staining in sham knees but increased staining in DMM knees from mice with reduced EGFR activity. The percentages of ADAMTS5- and MMP13-positive chondrocytes were quantified. $* P<0.05$; $* * *<0.001$. (c) qRT-PCR shows that EGFR signaling inhibits chondrogenic adamts5 expression. Primary chondrocytes were serum starved overnight, treated with vehicle (control) or TGF- $\alpha$ $\left(50 \mathrm{ng} \cdot \mathrm{mL}^{-1}\right.$ ) for 1 day, and harvested for mRNA quantification. $* * P<0.01$. 


\section{a}

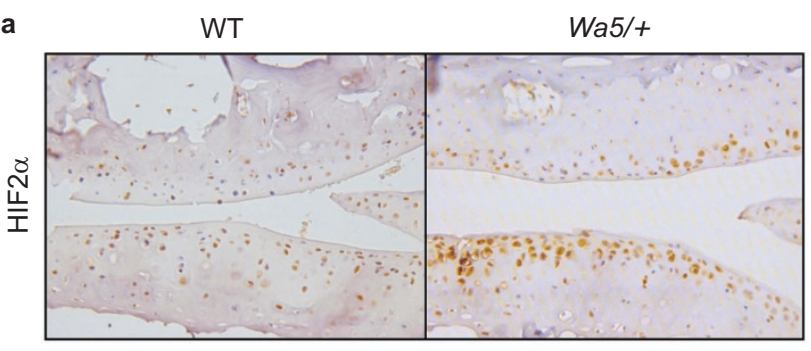

b

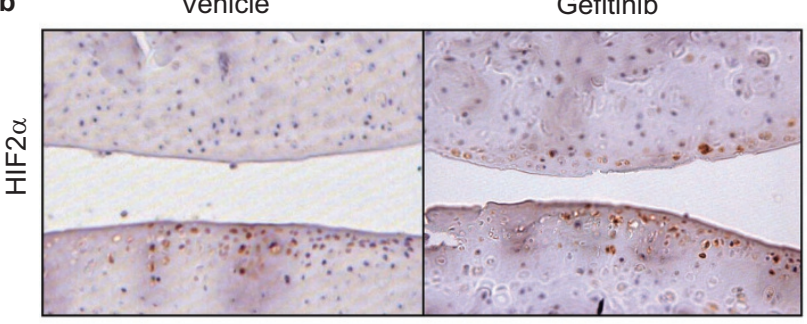

c

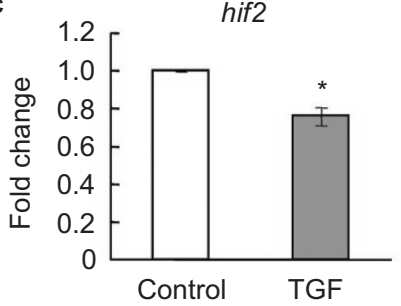

HIF $2 \alpha^{+}$
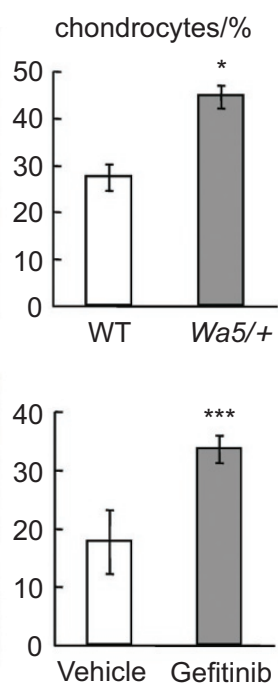

Figure 7. Deficiency of EGFR signaling increases HIF2 $\alpha$ amount in osteoarthritic cartilage. (a, b) Immunostaining of HIF2 $\alpha$ in DMM-operated knee joints from WT and Egfr ${ }^{W a 5 /+}$ mice $(\mathbf{a})$ and vehicle- and gefitinib-treated mice $(\mathbf{b})$. The percentage of HIF $2 \alpha$-positive chondrocytes was quantified $(n=4-$ 6 in each group). $* P<0.05$; *** $P<0.001$. (c) qRT-PCR shows that EGFR signaling inhibits chondrogenic hif $2 \alpha$ expression. Primary chondrocytes were serum starved overnight, treated with vehicle (control) or TGF- $\alpha\left(50 \mathrm{ng} \cdot \mathrm{mL}^{-1}\right)$ for 1 day, and harvested for mRNA quantification. * $P<0.05$.

catabolic proteinases (ADAMTS5 and MMP13) and a transcription factor (HIF2 $\alpha$ ) targeting cartilage matrix degradation after suppressing EGFR activity.

Our findings initially appeared surprising to us because they are not consistent with our previous conclusion that chondrogenic EGFR signaling stimulates cartilage ECM degradation by upregulating MMPs and stimulating osteoclastogenesis at the chondro-osseous junction at postnatal developmental stage. ${ }^{3}$ However, different from metabolically active epiphyseal chondrocytes at developmental stage, chondrocytes in adult cartilage are normally quiescent with very low activities of synthesizing and degrading cartilage matrix. In response to mechanical stress and pro-inflammatory cytokines, cartilage homeostasis is disrupted with increased ECM remodeling, hypertrophy-like maturation, cartilage calcification and chondrocyte apoptosis. Therefore, both anabolic and catabolic pathways are elevated during OA progression and imbalance between these two pathways toward more catabolic activity leads to this debilitating joint disease. Indeed, we and others have found that activating EGFR signaling executes both anabolic and catabolic actions on chondrocytes. It has been recognized for a long time that EGF is a potent mitogen for cultured chondrocytes. ${ }^{36-37}$ Interestingly, recent studies with either limb or cartilage targeted deletion of the intracellular EGFR inhibitor mig-6 revealed remarkably thickened articular cartilage in those mice at early adult stage due to enhanced chondrocyte proliferation. ${ }^{12-13}$ Although we did not notice a reduction in cartilage thickness in the sham-operated knees in mice with reduced EGFR activity, a close examination did reveal an increase in the number of hypertrophic chondrocytes, suggesting that EGFR prevents chondrocyte hypertrophy. Together with our data that EGFR signaling prevents chondrocyte apoptosis both in vitro and in vivo, these findings demonstrate a strong anabolic role of EGFR signaling in articular cartilage by increasing the number of articular chondrocytes, particularly proliferative and undifferentiated chondrocytes, which eventually leads to more cartilage matrix synthesis. Meanwhile, activating EGFR signaling transduces strong catabolic signals to chondrocytes, such as enhancing the expression of MMPs and RANKL ${ }^{3-4,9}$ and stimulating cartilage ECM degradation. ${ }^{3-4}$ Hence, we propose that the final outcomes in terms of OA progression in mouse with altered EGFR activity is combined effects of both anabolic and catabolic actions of EGFR pathway determined by the level of EGFR activity. According to this hypothesis, 
the severe OA phenotypes observed in mig-6 knockout mice is likely due to highly elevated EGFR activity. Note that our Egfr ${ }^{W 5 /+}$ mouse model has modest decrease in chondrogenic EGFR activity compared to WT mice while a chondrogenic EGFR knockout mouse model (col2-cre

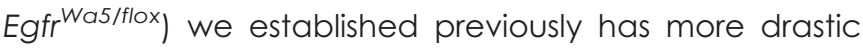
decrease in EGFR activity. ${ }^{6}$ We are currently studying the OA progression in this mouse model.

Excessive proteolysis has long been recognized as a major contributory factor to cartilage matrix degradation in OA. A variety of proteases have been implicated, ranging from lysosomal cysteine proteases to MMP family members. Among them, ADAMTS5 and MMP13 are the most critical ones in mediating cartilage ECM degradation because their expression is greatly enhanced in osteoarthritic knees ${ }^{38-41}$ and because mice deficient in either ADAMTS5 or MMP13 have diminished OA progression after DMM surgery. ${ }^{15-16,30,42}$ Interestingly, while ADAMTS5 amounts were similarly increased in both sham and DMM knee joints from mice with reduced EGFR activity, MMP13 amounts were decreased in sham knees, which is consistent with our previous report that EGFR signaling stimulates MMP13 expression in chondrocytes, but were increased in DMM knee joints from mice with reduced EGFR activity compared to their controls. The apparent contradiction could be explained by enhanced hif $2 \alpha$ expression in DMM knee joints after suppression of EGFR activity. Previous reports ${ }^{33-35}$ have shown that HIF $2 \alpha$ is a major regulator for Mmp 13 but not adamts 5 genes in chondrocytes. Elevated HIF2 $\alpha$ amount thus surpasses the other pathway(s) which mediates the stimulatory effect of EGFR signaling on $\mathrm{Mmp} 13$ expression.

In conclusion, in the present study, we provide the first direct evidence for the involvement of EGFR signaling in the OA development. Modestly decreased EGFR activity in our two mouse models accelerates articular cartilage destruction, which is associated with increased chondrocyte apoptosis and cartilage matrix degradation by ADAMTS5 and MMP13. This study lays the groundwork for understanding the role of EGFR and its cognate ligands in the maintenance of articular cartilage and in the pathogenesis of OA. Further studies to maximize its anabolic actions while minimizing its catabolic actions in cartilage will shed light on targeting this novel pathway in OA treatment.

\section{Competing interests}

The authors declare no conflict of interest.

\section{Acknowledgements}

The authors would like to thank John S. Mort at Shriners Hospital for Children (Montreal, Canada) for kindly providing antibodies for antiaggrecanase generated and anti-MMP generated aggrecan cleavage fragments. This study was supported by ASBMR Research Career
Enhancement Award (to LQ), NIH grants AR060991 (to LQ) and AR062908 (to ME-I)

\section{References}

1 Goldring MB, Goldring SR. Articular cartilage and subchondral bone in the pathogenesis of osteoarthritis. Ann NY Acad Sci 2010; 1192: 230-237.

2 Sharma AR, Jagga S, Lee SS, Nam JS. Interplay between cartilage and subchondral bone contributing to pathogenesis of osteoarthritis. Int $J$ Mol Sci 2013; 14: 19805-19830.

3 Zhang X, Siclari VA, Lan S et al. The critical role of the epidermal growth factor receptor in endochondral ossification. J Bone Miner Res 2011; 26: 2622-2633.

4 Usmani SE, Pest MA, Kim G, Ohora SN, Qin L, Beier F. Transforming growth factor alpha controls the transition from hypertrophic cartilage to bone during endochondral bone growth. Bone 2012; 51: 131141.

5 Wang K, Yamamoto H, Chin JR, Werb Z, Vu TH. Epidermal growth factor receptor-deficient mice have delayed primary endochondral ossification because of defective osteoclast recruitment. J Biol Chem 2004; 279: 5384853856

6 Zhang X, Zhu J, Li Y et al. Epidermal growth factor receptor (EGFR) signaling regulates epiphyseal cartilage development through betacatenin-dependent and -independent pathways. J Biol Chem 2013; 288: 32229-32240.

7 Sahin U, Weskamp G, Kelly K et al. Distinct roles for ADAM10 and ADAM17 in ectodomain shedding of six EGFR ligands. J Cell Biol 2004; 164: 769-779.

8 Hall KC, Hill D, Otero M et al. ADAM17 controls endochondral ossification by regulating terminal differentiation of chondrocytes. Mol Cell Biol 2013; 33: 3077-3090.

9 Saito K, Horiuchi K, Kimura Tet al. Conditional inactivation of TNFalphaconverting enzyme in chondrocytes results in an elongated growth plate and shorter long bones. PLoS One 2013; 8: e54853.

10 Appleton CT, Pitelka V, Henry J, Beier F. Global analyses of gene expression in early experimental osteoarthritis. Arthritis Rheum 2007; 56: $1854-1868$.

11 Appleton CT, Usmani SE, Bernier SM, Aigner T, Beier F. Transforming growth factor alpha suppression of articular chondrocyte phenotype and Sox9 expression in a rat model of osteoarthritis. Arthritis Rheum 2007; 56: 3693-3705.

12 Shepard JB, Jeong JW, Maihle NJ, O'Brien S, Dealy CN. Transient anabolic effects accompany epidermal growth factor receptor signal activation in articular cartilage in vivo. Arthritis Res Ther 2013; 15: R60.

13 Staal B, Williams BO, Beier F, Vande Woude GF, Zhang YW. Cartilagespecific deletion of Mig-6 results in osteoarthritis-like disorder with excessive articular chondrocyte proliferation. Proc Natl Acad Sci USA 2014; 111: 2590-2595

14 Zhang YW, Su Y, Lanning N et al. Targeted disruption of Mig-6 in the mouse genome leads to early onset degenerative joint disease. Proc Natl Acad Sci USA 2005; 102: 11740-11745.

15 Little CB, Barai A, Burkhardt D et al. Matrix metalloproteinase 13deficient mice are resistant to osteoarthritic cartilage erosion but not chondrocyte hypertrophy or osteophyte development. Arthritis Rheum 2009; 60: 3723-3733.

16 Wang M, Sampson ER, Jin H et al. MMP13 is a critical target gene during the progression of osteoarthritis. Arthritis Res Ther 2013; 15: R5.

17 Glasson SS, Blanchet TJ, Morris EA. The surgical destabilization of the medial meniscus (DMM) model of osteoarthritis in the 129/SvEv mouse. Osteoarthritis Cartilage 2007; 15: 1061-1069. 
18 Lee D, Cross SH, Strunk KE et al. Wa5 is a novel ENU-induced antimorphic allele of the epidermal growth factor receptor. Mamm Genome 2004; 15: 525-536.

19 Ma HL, Blanchet TJ, Peluso D, Hopkins B, Morris EA, Glasson SS. Osteoarthritis severity is sex dependent in a surgical mouse model. Osteoarthritis Cartilage 2007; 15: 695-700.

20 Aigner T, Cook JL, Gerwin N et al. Histopathology atlas of animal model systems-overview of guiding principles. Osteoarthritis Cartilage 2010; 18(Suppl 3): S2-S6.

21 Chandra A, Lan S, Zhu J, Siclari V, Qin L. Epidermal Growth Factor Receptor (EGFR) signaling promotes proliferation and survival in osteoprogenitors by increasing Early Growth Response Protein (Egr2) expression. J Biol Chem 2013; 288: 20488-20498.

22 Harari PM, Allen GW, Bonner JA. Biology of interactions: antiepidermal growth factor receptor agents. J Clin Oncol 2007; 25: 4057-4065.

23 Zhang X, Tamasi J, Lu X et al. Epidermal growth factor receptor plays an anabolic role in bone metabolism in vivo. J Bone Miner Res 2011; 26: 10221034.

24 Sharif M, Whitehouse A, Sharman P, Perry M, Adams M. Increased apoptosis in human osteoarthritic cartilage corresponds to reduced cell density and expression of caspase-3. Arthritis Rheum 2004; 50: 507515.

25 Thomas CM, Fuller CJ, Whittles CE, Sharif M. Chondrocyte death by apoptosis is associated with the initiation and severity of articular cartilage degradation. Int J Rheum Dis 2011; 14: 191-198.

26 D'Lima D, Hermida J, Hashimoto S, Colwell C, Lotz M. Caspase inhibitors reduce severity of cartilage lesions in experimental osteoarthritis. Arthritis Rheum 2006; 54: 1814-1821.

27 Malemud CJ, Islam N, Haqqi TM. Pathophysiological mechanisms in osteoarthritis lead to novel therapeutic strategies. Cells Tissues Organs 2003; 174: 34-48.

28 Heinegard D, Saxne T. Macromolecular markers in joint disease. J Rheumatol Suppl 1991; 27: 27-29.

29 Lohmander LS, Neame PJ, Sandy JD. The structure of aggrecan fragments in human synovial fluid. Evidence that aggrecanase mediates cartilage degradation in inflammatory joint disease, joint injury, and osteoarthritis. Arthritis Rheum 1993; 36: 1214-1222.

30 Glasson SS, Askew R, Sheppard B et al. Deletion of active ADAMTS5 prevents cartilage degradation in a murine model of osteoarthritis. Nature 2005; 434: 644-648.

31 Stanton H, Rogerson FM, East CJ et al. ADAMTS5 is the major aggrecanase in mouse cartilage in vivo and in vitro. Nature 2005; 434: $648-652$
32 Neuhold LA, Killar L, Zhao W et al. Postnatal expression in hyaline cartilage of constitutively active human collagenase-3 (MMP-13) induces osteoarthritis in mice. J Clin Invest 2001; 107: 35-44.

33 Hirata M, Kugimiya F, Fukai A et al. C/EBPbeta and RUNX2 cooperate to degrade cartilage with MMP-13 as the target and HIF2alpha as the inducer in chondrocytes. Hum Mol Genet 2012; 21: 11111123.

34 Saito T, Fukai A, Mabuchi A et al. Transcriptional regulation of endochondral ossification by HIF-2alpha during skeletal growth and osteoarthritis development. Nat Med 2010; 16: 678-686.

35 Tamiya H, Ikeda T, Jeong JH et al. Analysis of the Runx2 promoter in osseous and non-osseous cells and identification of HIF2A as a potent transcription activator. Gene 2008; 416: 53-60.

36 Gospodarowicz D, Mescher AL. A comparison of the responses of cultured myoblasts and chondrocytes to fibroblast and epidermal growth factors. J Cell Physiol 1977; 93: 117-127.

37 Kato Y, Gospodarowicz D. Growth requirements of low-density rabbit costal chondrocyte cultures maintained in serum-free medium. J Cell Physiol 1984; 120: 354-363.

38 Bau B, Gebhard PM, Haag J, Knorr T, Bartnik E, Aigner T. Relative messenger RNA expression profiling of collagenases and aggrecanases in human articular chondrocytes in vivo and in vitro. Arthritis Rheum 2002; 46: 2648-2657.

39 Flannelly J, Chambers MG, Dudhia J et al. Metalloproteinase and tissue inhibitor of metalloproteinase expression in the murine STR/ort model of osteoarthritis. Osteoarthritis Cartilage 2002; 10: 722-733.

40 Kevorkian L, Young DA, Darrah C et al. Expression profiling of metalloproteinases and their inhibitors in cartilage. Arthritis Rheum 2004; 50: 131-141.

41 Yang S, Kim J, Ryu JH et al. Hypoxia-inducible factor-2alpha is a catabolic regulator of osteoarthritic cartilage destruction. Nat Med 2010; 16: 687693.

42 Majumdar MK, Askew R, Schelling S et al. Double-knockout of ADAMTS-4 and ADAMTS- 5 in mice results in physiologically normal animals and prevents the progression of osteoarthritis. Arthritis Rheum 2007; 56: 3670-3674.

(c) (1) $(\Theta)$ This work is licensed under a Creative Commons Attribution(c) NonCommercial-NoDerivs 3.0 Unported License. The images or other third party material in this article are included in the article's Creative Commons license, unless indicated otherwise in the credit line; if the material is not included under the Creative Commons license, users will need to obtain permission from the license holder to reproduce the material. To view a copy of this license, visit http:/ / creativecommons.org/licenses/by-nc-nd/3.0/ 